\title{
The effect of natural and man-made disasters on countries' production efficiency
}

\author{
George Halkos ${ }^{1}$, Shunsuke Managi ${ }^{2}$ and Nickolaos G. Tzeremes ${ }^{*}$
}

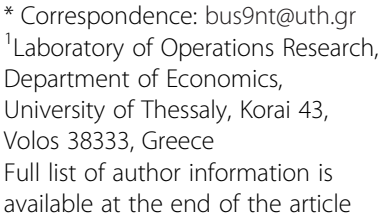

* Correspondence: bus9nt@uth.gr ${ }^{1}$ Laboratory of Operations Research, Department of Economics, University of Thessaly, Korai 43, Volos 38333, Greece

Full list of author information is available at the end of the article

\begin{abstract}
This study intends to understand how disaster is related to countries' production efficiency using a sample of 137 countries over 1980-2011. We analyze the effect of the number of man-made and natural disaster occurrences on countries' technological change (swift of the frontier) and technological catch-up (distribution of efficiencies). The results reveal an inverted " $U$ " shape relationship between countries' technological change and technological catch-up with disaster occurrences. This finding suggests that the effect on countries' production efficiency is positive for lower number of disaster events; however, after a specific threshold value, the effect becomes negative. The results also reveal that low-income countries are negatively affected much quicker compared to high-income countries. Finally, it is evident that the negative effect of disaster occurrences impacts first countries' technological catch-up and then their technology change.
\end{abstract}

JEL Classification: C14, D24, 047

Keywords: Disasters; Production efficiency; Conditional efficiency; Nonparametric analysis

\section{Background}

Disaster mitigation and adaptation are important for sustainable economy in a society. Macroeconomists and environmental economists have been studying on the economic analysis of disasters. The economic impact from disasters and the mechanism alleviating from disasters (such as insurance) has been well studied in the literature (see, e.g., Cavallo et al. 2013; McDermott et al. 2014). There are cases indicating that more disasters are associated with more GDP (Cavallo et al. 2013; Managi \& Sharma 2015). As has been pointed out by McDermott et al. (2014), mild shocks can stimulate reconstruction and therefore can cause growth. However, there is a broader view in the literature that humanitarian and climatic disasters have a direct negative effect on economic output (Raddatz 2007).

Often after a disaster event, an immediate request of an investment could be misused. This is because recovery from disasters requires immediate decision making which can result on less efficient use of investment to the economy. On the other hand, unused capital or old infrastructure could be replaced by new investment. This implies that there is a better chance of economic capitalization improvement, giving opportunities to new industries, by replacing old infrastructure. In this case, we expect that countries' production efficiency will improve after the disaster. Furthermore, it might be that the improvement in productivity is possible when disaster events are

\section{Springer}

(c) 2015 Halkos et al. This is an Open Access article distributed under the terms of the Creative Commons Attribution License (http:// creativecommons.org/licenses/by/4.0), which permits unrestricted use, distribution, and reproduction in any medium, provided the original work is properly credited. 
relatively small as recovery process is easily managed. In contrast when the disaster occurrences increase significantly, then there might be much less time for efficient decision making and as a consequence productivity decreases. If that is the case, an inverted " $U$ " shape relationship between productivity and disaster occurrences can be observed. However, another possibility is that productivity can be increased by a creative reconstruction when there are significant damages to the economy after an increased number of disaster occurrences. In addition, there is the possibility that for a small number of disaster occurrences (as well as for small disaster impacts), productivity might decrease since it is expected that there will be no change in industrial composition. In this case, a " $U$ " shape relationship between productivity and disaster occurrences could be seen from the data. This paper aims to provide empirical evidence examining such relationships in a global level. This is the first study which presents empirically the underline mechanism of how disaster is related to countries' productive efficiency and consequent productivity change using global data.

Specifically, our study contributes to the existing literature by investigating for the first time the effect of man-made and natural disaster occurrences on countries' production efficiency levels. Specifically, we apply time-dependent conditional efficiency measures in a nonparametric framework (Mastromarco \& Simar 2014). Then we utilize a newly developed approach introduced by Bădin et al. (2012) to a sample of 137 countries over the period of 1980-2011. In that respect, our paper examines in a dynamic framework the effect of disaster events on countries' technological change (swift of the frontier) and on countries' technological catch-up (distribution of efficiencies). Finally, our nonparametric framework does not assume any specific functional relationship of countries' production process and therefore provides us with the ability to reveal any nonlinear relationships which may exist.

The structure of our paper is as follows. Section 2 describes data and methodology while Section 3 presents the results. Finally, the last section concludes the paper.

\section{Methods}

\subsection{Data description}

For our methodological framework, we are using as countries' input factors capital stock at current PPPs (in millions 2005US\$) and labor force (in millions), whereas, our single output is the output-side real GDP at chained PPPs (in millions 2005US\$). Our data sample contains 137 countries (38 high-income, 24 upper-middle-income, 35 lower-middle-income, and 40 lower-income countries) ${ }^{1}$ over the period 1980-2011 (see "Appendix" for details). The input and output variables have been extracted from Penn World Table v8.0 (Feenstra et al. 2013). ${ }^{2}$

Moreover, the external variable affecting countries' production process is the number of man-made and natural disasters. ${ }^{3}$ The data on countries' disasters as a unit of disaster event number in each country are obtained from the Emergency Events Database (EM-DAT) from the Centre for Research on the Epidemiology of Disasters (CRED). ${ }^{4} \mathrm{Fi}$ nally, Table 1 below provides diachronically the descriptive statistics of the variables used. CRED collected each event for many countries over the long periods. As data collection starts from different year by country, it is unbalanced panel data. We choose our study periods to create balanced panel data to measure productivity. The database 
Table 1 Descriptive statistics of the variables used

\begin{tabular}{|c|c|c|c|c|c|c|c|c|c|c|c|}
\hline Year & Statistics & Total employment & Capital stock & GDP & Disasters & Year & Statistics & Total employment & Capital stock & GDP & Disasters \\
\hline \multirow[t]{2}{*}{1980} & Mean & 11.778 & $454,155.924$ & $168,126.344$ & 1.285 & 1996 & Mean & 16.480 & $800,107.820$ & $293,405.006$ & 3.029 \\
\hline & std & 47.038 & $1,739,488.751$ & $569,685.039$ & 2.755 & & std & 67.353 & $2,638,400.956$ & $959,456.060$ & 5.725 \\
\hline \multirow[t]{2}{*}{1981} & Mean & 12.127 & $471,044.931$ & $169,846.094$ & 1.197 & 1997 & Mean & 16.740 & $823,545.680$ & $302,636.873$ & 3.234 \\
\hline & std & 48.979 & $1,804,086.629$ & $580,843.148$ & 2.265 & & std & 68.243 & $2,725,033.235$ & $998,472.927$ & 7.221 \\
\hline \multirow[t]{2}{*}{1982} & Mean & 12.426 & $481,561.559$ & $169,356.021$ & 1.270 & 1998 & Mean & 16.995 & $848,505.233$ & $306,063.612$ & 3.606 \\
\hline & std & 50.617 & $1,842,351.213$ & $570,649.219$ & 2.858 & & std & 69.108 & $2,830,602.354$ & $1,028,573.363$ & 7.180 \\
\hline \multirow[t]{2}{*}{1983} & Mean & 12.683 & $468,443.433$ & $173,061.125$ & 1.635 & 1999 & Mean & 17.260 & $874,721.487$ & $317,639.343$ & 4.577 \\
\hline & std & 51.961 & $1,787,124.221$ & $591,469.221$ & 2.807 & & std & 69.926 & $2,945,857.958$ & $1,074,286.878$ & 7.804 \\
\hline \multirow[t]{2}{*}{1984} & Mean & 12.999 & $468,281.630$ & $181,575.919$ & 1.409 & 2000 & Mean & 17.558 & $910,560.729$ & $334,719.195$ & 5.628 \\
\hline & std & 53.319 & $1,794,357.103$ & $634,239.635$ & 2.977 & & std & 70.826 & $3,079,126.069$ & $1,126,005.233$ & 10.032 \\
\hline \multirow[t]{2}{*}{1985} & Mean & 13.334 & $474,792.230$ & $187,269.560$ & 1.628 & 2001 & Mean & 17.841 & $942,183.470$ & $340,233.218$ & 4.978 \\
\hline & std & 54.678 & $1,823,285.219$ & $658,474.221$ & 3.506 & & std & 71.793 & $3,216,026.326$ & $1,143,081.335$ & 9.617 \\
\hline \multirow[t]{2}{*}{1986} & Mean & 13.671 & $496,725.367$ & $194,929.618$ & 1.752 & 2002 & Mean & 18.117 & $972,784.272$ & $348,121.142$ & 5.606 \\
\hline & std & 55.936 & 1,890,802.076 & $682,741.112$ & 3.925 & & std & 72.788 & $3,344,003.740$ & $1,165,718.655$ & 10.043 \\
\hline \multirow[t]{2}{*}{1987} & Mean & 14.000 & $517,069.651$ & $203,732.140$ & 2.438 & 2003 & Mean & 18.432 & $1,025,260.303$ & $358,278.790$ & 4.599 \\
\hline & std & 57.065 & $1,950,505.109$ & $707,574.290$ & 4.794 & & std & 73.714 & $3,505,394.653$ & $1,205,161.938$ & 9.295 \\
\hline \multirow[t]{2}{*}{1988} & Mean & 14.343 & $542,888.825$ & $212,644.927$ & 2.482 & 2004 & Mean & 18.809 & $1,115,236.401$ & $376,384.210$ & 4.635 \\
\hline & std & 58.494 & $2,024,926.264$ & $737,002.408$ & 5.775 & & std & 74.864 & $3,765,697.494$ & $1,258,918.758$ & 9.762 \\
\hline \multirow[t]{2}{*}{1989} & Mean & 14.701 & $567,677.451$ & $219,678.706$ & 2.569 & 2005 & Mean & 19.101 & $1,228,107.151$ & $398,768.660$ & 5.328 \\
\hline & std & 60.361 & $2,092,597.704$ & $761,853.568$ & 5.488 & & std & 75.649 & $4,114,511.020$ & $1,317,702.953$ & 11.114 \\
\hline \multirow[t]{2}{*}{1990} & Mean & 15.062 & $598,391.882$ & $228,252.970$ & 3.204 & 2006 & Mean & 19.449 & $1,362,797.584$ & $414,236.223$ & 4.737 \\
\hline & std & 62.023 & $2,153,172.393$ & $780,555.343$ & 6.204 & & std & 76.563 & $4,509,109.447$ & $1,370,650.023$ & 9.209 \\
\hline \multirow[t]{2}{*}{1991} & Mean & 15.321 & $616,338.768$ & $233,729.672$ & 3.241 & 2007 & Mean & 19.802 & $1,462,579.240$ & $433,107.615$ & 4.467 \\
\hline & std & 63.383 & $2,183,997.256$ & $787,548.430$ & 6.268 & & std & 77.473 & $4,770,668.327$ & $1,425,377.191$ & 7.398 \\
\hline
\end{tabular}


Table 1 Descriptive statistics of the variables used (Continued)

\begin{tabular}{|c|c|c|c|c|c|c|c|c|c|c|c|}
\hline \multirow[t]{2}{*}{1992} & Mean & 15.549 & $635,992.718$ & $244,081.059$ & 2.343 & 2008 & Mean & 20.095 & $1,552,115.359$ & $440,736.543$ & 4.248 \\
\hline & std & 64.249 & $2,216,966.358$ & $818,800.869$ & 4.806 & & std & 78.203 & $5,019,146.147$ & $1,444,895.526$ & 7.040 \\
\hline \multirow[t]{2}{*}{1993} & Mean & 15.750 & $671,855.674$ & $254,104.209$ & 2.723 & 2009 & Mean & 20.265 & $1,579,023.191$ & $441,339.897$ & 3.839 \\
\hline & std & 65.083 & 2,309,684.885 & $850,061.215$ & 6.276 & & std & 78.840 & $5,115,717.649$ & 1,436,698.346 & 6.545 \\
\hline \multirow[t]{2}{*}{1994} & Mean & 16.011 & $714,960.881$ & $266,518.840$ & 2.781 & 2010 & Mean & 20.570 & $1,623,685.784$ & $463,966.400$ & 4.088 \\
\hline & std & 65.862 & 2,421,837.193 & $889,099.612$ & 5.620 & & std & 79.588 & $5,287,237.525$ & $1,507,251.764$ & 6.901 \\
\hline \multirow[t]{2}{*}{1995} & Mean & 16.210 & $767,889.398$ & $280,462.926$ & 2.766 & 2011 & Mean & 20.873 & $1,713,752.560$ & $479,023.024$ & 3.898 \\
\hline & std & 66.541 & $2,565,239.496$ & $923,961.730$ & 4.837 & & std & 80.302 & $5,658,061.263$ & $1,567,260.611$ & 6.750 \\
\hline
\end{tabular}


is part of our comprehensive database called World Resource Table (WRT) (see Miyama \& Managi 2014; Managi 2015). WRT includes all related data and intends to update once new version available (Yang et al. 2015).

\subsection{Methodology}

In order to measure a country's production efficiency, let $X \in \Re_{+}^{p}$ and $Y \in \Re_{+}^{q}$ be the input and output vectors, representing total labor force and capital stock the inputs and GDP the outputs. Furthermore, let $Z \in \Re^{d}$ be the vector of the environmental variable (the number of man-made and natural disaster occurrences). We can define the unconditional attainable set as:

$$
\Psi=\left\{(x, y) \in \mathfrak{R}_{+}^{p+q} \mid x \text { can produce } y\right\},
$$

which according to Daraio and Simar $(2005 ; 2007 a)$ can also be characterized as:

$$
\begin{gathered}
\Psi=\left\{(x, y) \mid H_{X, Y}(x, y)>0\right\} \text { where } \\
H_{X, Y}(x, y)=\operatorname{Prob}(X \leq x, Y \geq y) .
\end{gathered}
$$

Then the output oriented Farell-Debreu technical efficiency can be defined as:

$$
\lambda(x, y)=\sup \{(\lambda \mid x, \lambda y) \in \Psi\}=\sup \left\{\lambda \mid S_{Y \mid X}(\lambda y \mid x)>0\right\},
$$

where $S_{Y \mid X}(y \mid x)=\operatorname{Prob}(Y \geq y \mid X \leq x)$. Mastromarco and Simar (2014) suggested that dynamic effects can be captured when incorporating time $T$ as an extra conditional variable along side with $Z$ (i.e., the number of man-made and natural disaster occurrences). Then the attainable set can be defined as $\Psi_{t}^{z} \subseteq \Psi_{t} \subset \mathfrak{R}_{+}^{p+q}$, and its distribution can be characterized as:

$$
H_{X, Y \mid Z}^{t}(x, y \mid z)=\operatorname{Prob}(X \leq x, Y \geq y \mid Z=z, T=t) .
$$

Then a country's conditional output oriented technical efficiency level $(x, y) \in \Psi_{t}^{z}$ at time $t$ facing the effect of disasters $z$ can be defined as:

$$
\lambda_{t}(x, y \mid z)=\sup \left\{\lambda \mid S_{Y \mid X, Z}^{t}(\lambda y \mid x, z)>0\right\} .
$$

Moreover, based on Daouia and Simar (2007), the robust versions of the production efficiency estimates can be calculated (known as Order- $\alpha$ quantile efficiency measure) for any $\alpha \in(0,1)$ as:

$$
\lambda_{\alpha}(x, y)=\sup \left\{\lambda \mid S_{Y \mid X}(\lambda y \mid x)>1-\alpha\right\} .
$$

Then the time-dependent conditional Order- $\alpha$ quantile efficiency measure can be defined as:

$$
\lambda_{t, \alpha}(x, y \mid z)=\sup \left\{\lambda \mid S_{Y \mid X, Z}^{t}(\lambda y \mid x, z)>1-\alpha\right\},
$$

where $S_{Y \mid X, Z}^{t}(y \mid x, z)=\operatorname{Prob}(Y \geq y \mid X \leq x, Z=z, T=t)$. According to Daraio and Simar (2007b), robust efficiency measures are not so sensitive to outliers and they are not suffering from the curse of dimensionality. Furthermore, the conditional efficiency measures ${ }^{5}$ presented in (5) and (6) assume that the separability condition between the inputs, outputs, the time, and the disasters does not hold, implying that time and the number of disaster occurrences influence directly the shape (the boundary) of the attainable set. ${ }^{6}$

In order to calculate the original and the time-dependent conditional efficiency scores, we apply data envelopment analysis (DEA) estimators. In Equations 1 and 3, we 
assume variable returns to scale (VRS) to account for size differences among countries. Whereas for the robust frontiers (Equations 4 and 5), we follow the suggestion made by Bădin et al. (2012) and we apply a median quartile $(\alpha=0.5)$ rather than the extreme quartiles $(\alpha=0.9, \alpha=0.95)$. This in turn will enable us to investigate the effect of time and the number of disaster occurrences on the distribution of inefficiencies. In contrast, the analysis with the full frontiers enables us to investigate the effect of time and the number of disaster occurrences on the frontier. ${ }^{7}$

Finally, by following Bădin et al. (2012), we construct two ratios from the efficiencies calculated from the full and the partial frontiers as:

$$
\begin{gathered}
Q=\frac{\lambda_{t}(x, y \mid z)}{\lambda(x, y)}, \\
Q_{\alpha}=\frac{\lambda_{t, \alpha}(x, y \mid z)}{\lambda_{\alpha}(x, y)} .
\end{gathered}
$$

Then by applying a local constant nonparametric regression on the above ratios, we can determine the effect of time and the number of disaster occurrences on the boundary-swift of the frontier (for the case of full frontier: $Q$ ) and on the distributions of countries' efficiencies (for the case of partial frontier: $Q_{\alpha}$ ). For our analysis, we are using local constant techniques, and for bandwidth selection, we apply the least squares cross-validation (LSCV) criterion as described in (Hall et al. 2004; Li \& Racine 2007). An increasing regression line indicates a positive effect on countries' technological change (shift on the frontier) and on the distribution of their efficiencies (technological catch-up). However, the opposite indicates an unfavorable effect.

\section{Results and discussion}

Figure 1 illustrates graphically countries' production efficiency curves both for conditional and unconditional cases over the period 1980-2011. ${ }^{8}$ The results presented are based on countries' income classification and are obtained under the assumption of variable returns to scale (VRS). ${ }^{9}$ Figure 1a examines the original production efficiency scores over the examined period. It indicates that there was a trend towards similar levels of production efficiencies for high-income and upper-medium-income countries over the period 1980 to 1996.
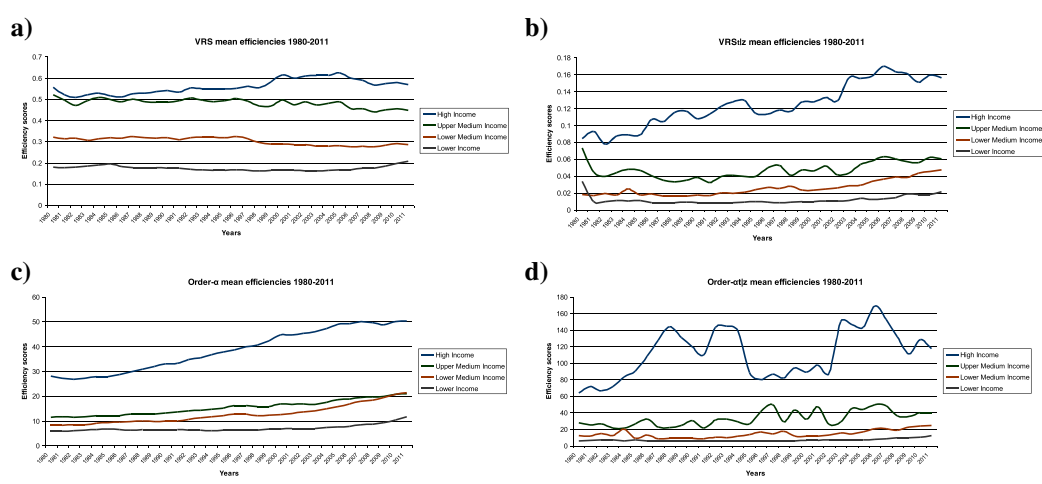

Fig. 1 a-d Countries' mean production efficiency estimates for 1980-2011 based on income levels 
However, after this point, there is evidence that countries' production efficiency levels divert. A similar phenomenon is also observed for the larger period for lower-mediumand lower-income countries. However, after 2007, it appears that there is a trend towards similar efficiency levels. Figure 1c presents countries' the original production efficiency estimates of the Order- $\alpha$ frontier. Since these estimates are not bounded by 1, then we observe extremely high efficiency scores compared to the full frontiers (i.e., VRS frontier in our case).

The results show that high-income countries have steadily increased their efficiency estimates over the years with a production efficiency stabilization period from 2007 to 2011. This might be attributed due to the initiation of Global Financial Crisis. Furthermore, as can be observed, there is a trend of similar production efficiency levels for upper-medium and lower-medium income countries (especially over the period 20062011), whereas lower-income countries appear to increase their production efficiency levels over the years but with lower estimated efficiency scores compared to the other three income groups.

Furthermore, Fig. 1b, d presents diachronically countries' efficiency curves when accounting both for the number of disaster occurrences and time (i.e., time-dependent conditional efficiency estimates). For the case of full frontiers (Fig. 1b), we can find that high-income countries have greater efficiency scores compared to the other three groups. It is also observed that under the effect of time and the number of disaster occurrences, lower-medium and lower-income countries' production efficiencies have similar trend from 1980s to early 1990s. However, after that point, a trend of similar efficiency levels is observed between upper-medium- and lower-medium- income countries, whereas the group of lower-income countries seems to divert in terms of their production efficiency levels. For the case of robust measures (Fig. 1d), again it is observed that the group of high-income countries has significant higher time-dependent conditional efficiency scores compared to the other three groups. Finally, when examining the group of high-income countries both for the full (Fig. 1b) and partial frontier (Fig. 1d), we observe that during the 1990s, their efficiency levels have decreased. This phenomenon is more pronounced when looking the conditional robust estimators. Since these two graphs account directly for the effect of time (alongside with the effect of the number of disaster events), our finding supports the findings by Marone (2009) that during the 1990s, high-income countries have decreased their growth rates. This in turn reflects upon their conditional efficiency estimates. Regardless, the efficiency gap between high-income countries and the other groups remains. Furthermore, the trend of similar levels of production efficiencies is observed between lower-mediumand lower-income countries. Clearly we can observe from Fig. 1 that when we account both for the effect of time and disasters, countries' production efficiency curves seem to be highly fluctuated, this is especially more intense for the case of high-income countries.

In addition to the previous finding, Fig. 2 examines both the time-dependent conditional and unconditional efficiency estimates of the seven major economies (known also as G7) both under the full and robust frontiers. Specifically under the full frontier (Fig. 2a), USA is reported as the top performer. It seems that there was a similar level of efficiencies with the GBR at early 2000s, but after that period, a diversion of efficiencies is reported. Furthermore, it seems that DEU, FRA, ITA, and GBR have similar 
a)

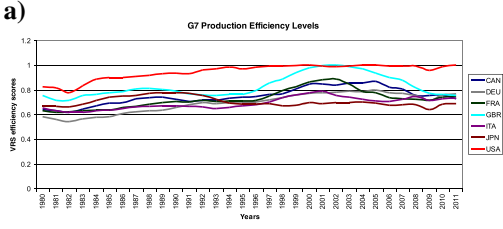

c)

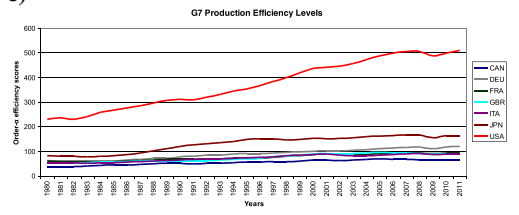

b)

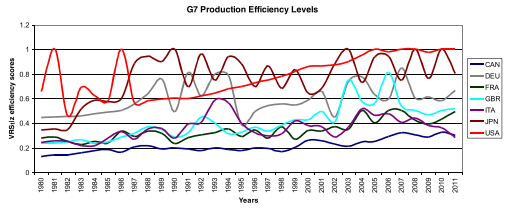

d)

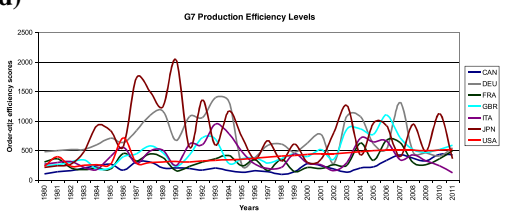

Fig. 2 a-d G7 Countries' mean production efficiencies estimates for 1980-2011

production efficiency levels especially during the beginning of Global Financial Crisis (i.e., 2008 to 2011), with JPN to divert slightly from that group. Under the robust frontier analysis (Fig. 2c), the results are different.

USA again outperforms the other countries with an increasing efficiency trend up to the beginning of Global Financial Crisis. Then the second performer is JPN, whereas, it appears the existence of similar efficiency levels among the European countries (DEU, FRA, GBR, and ITA). Finally, the last performer is CAN which seems to divert from the European group. Figure $2 \mathrm{~b}, \mathrm{~d}$ is presented here only for the purpose of comparison and to demonstrate how the effects of time and disasters influence the largest economies' production efficiency curves. In both cases (i.e., for the full and robust frontiers) and in comparison with the original estimates, it is observed that G7 countries' efficiency curves are highly fluctuated under the effect of time and disaster events presenting a highly nonlinear path.

There is only one exception and that is the case of the USA, which appears to have a fairly steady increasing production efficiency path in the longer part of the examined period. This indicates that the USA responds better to man-made and natural disasters compare to the other economies (with the exception over the period 1980 to 1987).

In order to investigate the effect of time and disasters on countries' production efficiency, we follow the approach by Bădin et al. (2012) by presenting the threedimensional pictures of the joint effect. The left-hand side (Fig. 3a, c, e, g, and i) on Fig. 3 represents the effect of time and the number of disaster occurrences on countries' technological change (shifts in the frontier), whereas the right-hand side (Fig. 3b, d, f, h, and j) represents the effect on countries' efficiency levels (technological catch-up). The overall picture suggests that there is a nonlinear relationship between the number of disaster occurrences and countries' productive efficiency levels. Specifically, when examining the entire sample (Fig. 3a, b), an inverted " $U$ " shape between countries' technological change and technological catch-up with disasters is revealed.

This finding suggests that lower number of disasters tend to increase countries' technological change and technological catch-up up to a certain point. After that point (threshold number), the effect is negative. The positive effect maybe attribute to the fact that for lower number of disasters, countries engage restructuring and investment, which in turn accelerates both their technological change and technological catch-up levels. In fact, this finding is in the same lines with Skidmore and Toya (2002) suggesting 


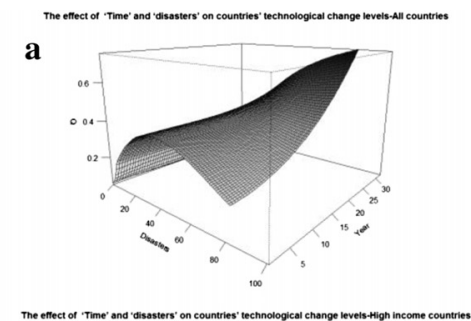

c

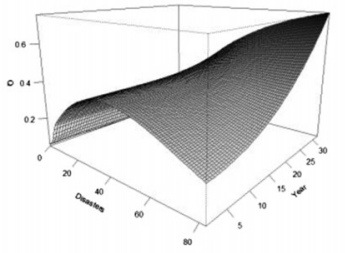

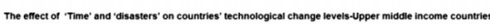

e

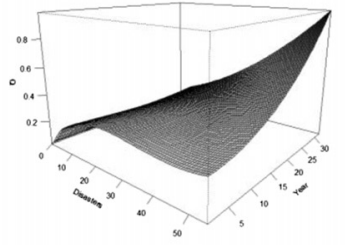

g

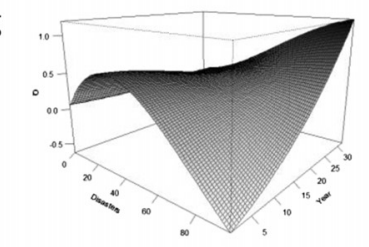

i

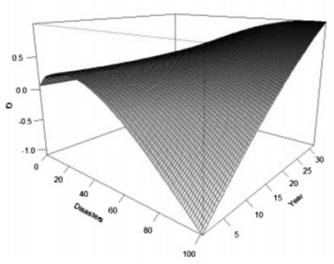

b

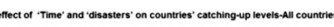

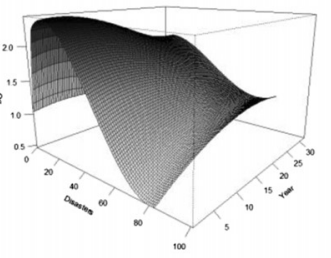

d

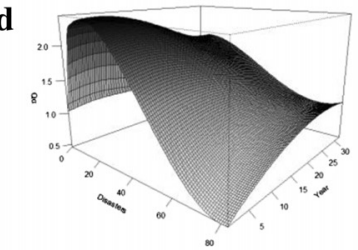

f

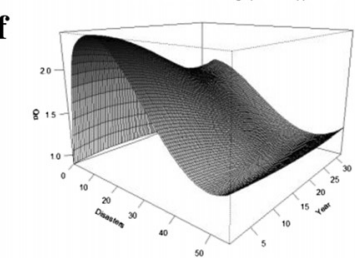

h
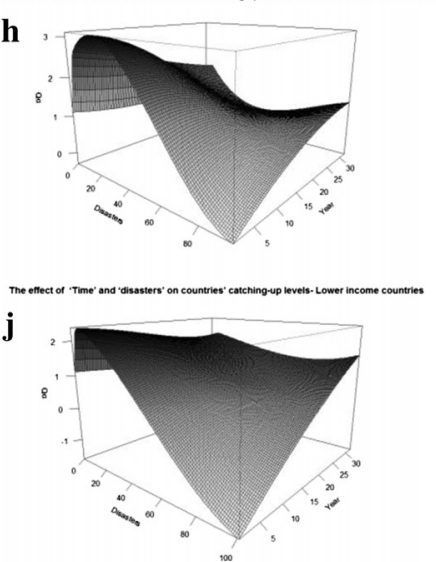

Fig. $\mathbf{3} \mathbf{a}-\mathbf{j}$ The effect of disasters and time on countries' production efficiency levels

that the disaster occurrences are positively correlated with economic growth. Other studies (McDermott et al. 2014; Raddatz 2007; Noy 2009) suggest that the overall effect of disasters is negative; however, mild disaster shocks can stimulate reconstruction activity and can impact positively countries' economic growth. In our case, the negative influence maybe attribute due to the fact that a large number of disaster occurrences or small number of disasters with high impact can have a negative impact on countries' production factors which reflects on their technological change and technological catch-up levels. Also it must be mentioned that the threshold value in which the influence from positive becomes negative is much lower for the case of technological catch-up (i.e., 15 disaster occurrences) compared to technological change (i.e., 25 disaster occurrences). This suggests that the negative influence of the number of disaster occurrences first affects countries' production efficiency and then their technological change levels. The effect of time is positive for the 
case of technological change indicated by an increasing nonparametric line. For the case of technological catch-up, the effect is positive for the larger period; however, towards the Global Financial Crisis, the effect becomes rather neutral on countries' technological catchup levels. Finally, it must be emphasized that according to McDermott et al. (2014), the combination of the type and severity of the events along side with countries' socioeconomic characteristics is very crucial and can shape the effect of disasters on countries' efficiency levels. ${ }^{10}$ In our case and in order to account for countries' different economic characteristics, we continue our analysis in a similar manner into four subsamples which are based on their income characteristics (high-income countries (Fig. 3c, d); upper-middle-income countries (Fig. 3e, f); lower-middle-income countries (Fig. 3g, h); lower-income countries (Fig. 3i, j)).

It is evident that regardless of countries' different income levels, the effect of time and the number of disasters on countries' technological catch-up and technological change is similar compared to our previous findings. However, an interesting observation is that the turning points (i.e., the threshold values) which the effect turns form positive to negative both for countries' technological catch-up and technological change is based on countries' income level. For instance, when examining the group of highincome countries, we can observe that the turning point of the effect on technological change (Fig. 3c) is 39 disaster occurrences, whereas, of the effect on technological catch-up (Fig. 3d) is 20 disaster occurrences. However, for the upper-middle-income countries (Fig. 3e, f), the critical points are indicated for 12 and 10 disaster occurrences, for lower-middle-income countries (Fig. 3g, h) for 20 and 17 disaster occurrences, and finally, for lower-income countries (Fig. 3i, j) for 20 and 8 disaster occurrences. This finding suggests that higher-income countries have higher turning points compared to the rest of the country groups. This can be attributed to the fact that lower-income countries' are not able to respond to disasters in the same way as high-income countries, and as a result, the negative effect comes quicker for those countries. Noy (2009) provides evidence that the disaster effects are more severe for countries with a weak financial sector. Therefore, since high-income countries have stronger financial sectors compared to the lowerincome countries, the negative effect of disaster occurrences impacts first the lowerincome countries. Moreover, it is also evident that for all the country groups, the turning points for the effect on technological catch-up are much lower compared to the turning points of the effects on technological catch-up. This finding as has been mentioned previously implies that regardless of the income level of a country, the negative effect of disaster occurrences impacts first country's ability to catch-up and then its technology change.

Finally, when examining the effect of time, it is observed that in most of the cases, the influence is positive but in a nonlinear manner. Two exceptions can be observed for the case of upper-middle- and high-income countries' technological catch-up (Fig. 3f, d), in which the effect of time is neutral especially during the initiation period of Global Financial Crisis. ${ }^{11}$

\section{Conclusions}

We examine the effect of the number of man-made and natural disaster occurrences on countries' production efficiency levels in a fully nonparametric framework to a sample of 137 countries over the period 1980-2011. Specifically by applying the newly 
developed time-dependent conditional efficiency measures by Mastromarco and Simar (2014) and the methodological approach by Bădin et al. (2012), we examine the effect of disaster occurrences on countries' technological change and technological catch-up. To our knowledge, this is the first study that examines the relationship between disasters and countries' production efficiency in a fully nonparametric framework.

The empirical findings suggest that the relationship is nonlinear forming an inverted "U" shape both with countries' technological change and technological catch-up. Furthermore, the results suggest that this inverted " $U$ " shape relationship is revealed regardless of countries' income classification. Moreover, the results indicate that a lower number of disaster occurrences have a positive influence on countries technological change and technological catch-up due to possible stimulation of restructuring and investment policies imposed by governments as counteractions of those events.

However, it is also evident that after a certain threshold level (i.e., certain number of disaster occurrences), the effect becomes negative influencing countries' production factors. Since the study investigates the effect of the number of disaster occurrences (and not the effect generated by the specific type and severity of the disasters), when the number of disaster occurrences increases, then the probability of having high impact disasters within those occurrences also increases. This in turn indicates a clear negative effect on countries' technological change and technological catch-up levels. Furthermore, our findings suggest that the turning points are much lower for lowerincome countries indicating that these countries suffer more from the negative impact of disasters. Finally, it is also shown that regardless of countries' income classification, the negative effect of the number of disaster occurrences influences first countries' efficiency and then their technological change levels.

Finally, an extension of our work which is left for future research can be focused on the examination of specific types of natural and man-made disasters on countries' production process controlling also for countries' differences on their industrial compositions and alongside with other country-specific characteristics (i.e., countries' institutional quality). From the methodological point of view and in the framework of nonparametric frontier analysis, the proposed future research can be approached either by double bootstrap semi-parametric approaches (Simar \& Wilson 2007) or by the more flexible conditional directional distance functions (Simar \& Vanhems 2012; Daraio \& Simar 2014).

\section{Endnotes}

${ }^{1}$ The income classification is based on IMF, which is available from http://www.imf.org/external/pubs/ft/weo/2014/02/weodata/weoselgr.aspx

${ }^{2}$ According to (Johnson et al. 2013), the previous PWT versions have suffered from valuation problems. However, according to (Feenstra et al. 2013), the new version of PWT (v8.0) is more consistent over time and more transparent in its methods. The data is available from www.ggdc.net/pwt.

${ }^{3}$ The events from natural disasters contain climatic disasters (i.e., drought, extreme temperatures, floods, storms, mass movement wet and wild fire), geologic disasters (i.e., earthquakes and volcanic eruptions) and biologic disasters (i.e., epidemics disasters and disasters derived from insects). The events from man-made disasters contain complex disasters, industrial accidents, miscellaneous accidents, and transport accidents. 
${ }^{4}$ The data are available from http://www.emdat.be/database.

${ }^{5}$ Jeong et al. (Jeong et al. 2010) provide the asymptotic properties of conditional efficiency measures.

${ }^{6}$ For details on the subject matter, see Simar and Wilson (2007).

${ }^{7}$ For in depth analysis regarding the smoothing techniques, bandwidth selection, kernel choices, and LP programs, see the works of Daraio and Simar (2005; 2007a; 2007b), Bădin et al., (2012; 2010) and Mastromarco and Simar (2014).

${ }^{8}$ Due to the enormous quantity of the results obtained, it is not feasible to present the analytical (per country) efficiency estimates both for conditional and unconditional frontiers both for full and their robust versions. However, all country estimates are available upon request.

${ }^{9}$ The VRS assumption has been chosen in order to account in our analysis the scale effects which are present between countries. Furthermore and in order to compare countries' with similar characteristics, we have grouped our sample based on Worlds Bank's income classification levels. Therefore, in our analysis, we have four categories: high-income countries, upper-medium-income countries, lower-medium-income countries, and lower-income countries.

${ }^{10}$ In our study, we do not account for the effect generated by the specific type and severity of the disasters but only for the effect generated by the number of disaster occurrences. In that respect, the results presented must be interpreted with extra care.

${ }^{11}$ Similar results are also reported by Tzeremes (2014).

\section{Appendix: Country list used in this study}

High-income countries (38): AUS, AUT, BEL, BHR, BHS, BRB, BRN, CAN, CHE, CYP, DEU, DNK, ESP, FIN, FRA, GBR, GRC, HKG, IRL, ISL, ISR, ITA, JPN, KOR, KWT, LUX, MAC, MLT, NLD, NOR, NZL, PRT, QAT, SAU, SGP, SWE, TTO, USA

Upper-middle-income countries (24): ARG, BGR, BLZ, BRA, BWA, CHL, CRI, GAB, GNQ, HUN, LBN, LCA, MEX, MUS, MYS, OMN, PAN, POL, TUR, TWN, URY, VCT, VEN, ZAF.

Lower-middle-income countries (35): AGO, ALB, BOL, BTN, CHN, CMR, COD, COL, CPV, DJI, DOM, ECU, EGY, FJI, GTM, HND, IDN, IRN, IRQ, JAM, JOR, LKA, LSO, MAR, MDV, NAM, PER, PHL, PRY, SLV, SUR, SWZ, SYR, THA, TUN.

Lower-income countries (40): BDI, BEN, BFA, BGD, CAF, CIV, COG, COM, ETH, GHA, GIN, GMB, GNB, IND, KEN, KHM, LAO, LBR, MDG, MLI, MNG, MOZ, MRT, MWI, NER, NGA, NPL, PAK, RWA, SDN, SEN, SLE, STP, TCD, TGO, TZA, UGA, VNM, ZMB, ZWE. 
Received: 17 April 2015 Accepted: 16 June 2015

Published online: 30 July 2015

\section{References}

Bădin L, Daraio C, Simar L (2010) Optimal bandwidth selection for conditional efficiency measures: a data-driven approach. Eur J Oper Res 201:633-640

Bădin L, Daraio C, Simar L (2012) How to measure the impact of environmental factors in a nonparametric production model? Eur J Oper Res 223:818-833

Cavallo E, Galiani S, Noy I, Pantano J (2013) Catastrophic natural disasters and economic growth. Rev Econ Stat 95(5):1549-1561

Daouia A, Simar L (2007) Nonparametric efficiency analysis: a multivariate conditional quantile approach. J Econometrics 140:375-400

Daraio C, Simar L (2005) Introducing environmental variables in nonparametric frontier models: a probabilistic approach. J Prod Anal 24:93-121

Daraio C, Simar L (2007a) Advanced robust and nonparametric methods in efficiency analysis. Methodology and applications, Springer, New York

Daraio C, Simar L (2007b) Conditional nonparametric frontier models for convex and non convex technologies: a unifying approach. J Prod Anal 28:13-32

Daraio C, Simar L (2014) Directional distances and their robust versions: computational and testing issues. Eur J Oper Res 16:358-369

Feenstra RC, Inklaar R, Timmer MP (2013) The next generation of the Penn World Table., Available for download at www.ggdc.net/pwt

Hall P, Racine JS, Li Q (2004) Cross-validation and the estimation of conditional probability densities. J Am Stat Assoc 99:1015-1026

Jeong S, Park B, Simar L (2010) Nonparametric conditional efficiency measures: asymptotic properties. Annals of Operational Research 173(1):105-122

Johnson S, Larson W, Papageorgiou C, Subramanian A (2013) Is newer better? Penn World Table revisions and their impact on growth estimates. J Monet Econ 60:255-274

Li Q, Racine J (2007) Nonparametric econometrics: theory and practice. Princeton University Press, New Jersey, USA

Managi S (ed) (2015) The Routledge Handbook of Environmental Economics in Asia. Routledge, New York, USA

Managi S, Sharma S (2015) Economics of disasters: empirical analysis. The Singapore Economic Review (forthcoming)

Marone $\mathrm{H}$ (2009) Economic growth in the transition from the 20th to the 21st century. UNDP/ODS Working Paper,

Office of Development Studies United Nations Development Programme, New York, Available from: http:// web.undp.org/developmentstudies/docs/economic_context_oct2009.pdf

Mastromarco C, Simar L, 2014. Effect of FDI and time on catching-up: new insights from a conditional nonparametric frontier analysis. J Appl Econom. doi: 10.1002/jae.2382.

McDermott TKJ, Barry F, Tol RSJ (2014) Disasters and development: natural disasters, credit constraints and economic growth. Oxf Econ Pap 66(3):750-773

Miyama E, Managi S (2014) Global environmental emissions estimate: application of multiple imputation. Environmental Economics and Policy Studies 16(2):115-135

Noy I (2009) The macroeconomic consequences of disasters. J Dev Econ 88:221-31

Raddatz C (2007) Are external shocks responsible for the instability of output in low income countries? J Dev Econ 84:155-87

Simar L, Vanhems A (2012) Probabilistic characterization of directional distances and their robust versions. J Econ 166:342-354

Simar L, Wilson PW (2007) Estimation and inference in two-stage, semi-parametric models of production processes. Econ 136:31-64

Skidmore M, Toya H (2002) Do natural disasters promote long-run growth? Econ Inq 40:664-87

Tzeremes NG (2014) The effect of human capital on countries' economic efficiency. Econ Lett 124:127-131

Yang J, Managi S, Sato M (2015) The effect of institutional quality on national wealth: an examination using multiple imputation method, environmental economics and policy studies.. doi:10.1007/s10018-014-0084-z

\section{Submit your manuscript to a SpringerOpen ${ }^{\circ}$ journal and benefit from:}

- Convenient online submission

Rigorous peer review

- Immediate publication on acceptance

- Open access: articles freely available online

- High visibility within the field

Retaining the copyright to your article 